\title{
Study of the LISM Using Pulsar Scintillation
}

\author{
N.D.R. Bhat, Y. Gupta, and A.P. Rao \\ National Centre for Radio Astrophysics (TIFR), Pune 411 007, India.
}

\begin{abstract}
We present here the results from an extensive scintillation study of twenty pulsars in the dispersion measure (DM) range $3-35 \mathrm{pc} \mathrm{cm}^{-3}$ carried out using the Ooty Radio Telescope, to investigate the distribution of ionized material in the local interstellar medium (LISM). Our analysis reveals several anomalies in the scattering strength, which suggest that the distribution of scattering material in the solar neighborhood is not uniform. Our model suggests the presence of a low density bubble surrounded by a shell of much higher density fluctuations. We are able to put some constraints on geometrical and scattering properties of such a structure, and find it to be morphologically similar to the local bubble known from other studies.
\end{abstract}

\section{Introduction}

Propagation effects on pulsar signals, such as dispersion and scattering, probe the distribution of thermal plasma in the interstellar medium (ISM). Scattering studies of radio waves from pulsars, enable us to probe the electron density fluctuations in the ISM, which are presumably due to turbulence in the ISM (Rickett 1990). Not much is known about electron densities and their fluctuations in the LISM and in order to investigate this in detail, we have made extensive scintillation observations of twenty nearby pulsars. Reliable and accurate estimates of strength of scattering have been obtained and the results are used to study the distribution of electron density fluctuations in the LISM.

\section{Observations and Data Analysis}

The observations were made using the Ooty Radio Telescope (ORT) at 327 MHz. Within the sky coverage and sensitivity limits of the ORT, there are twenty nearby pulsars that were found suitable for studying the LISM. For these pulsars, the dynamic scintillation spectra - intensity variation in the frequency-time plane - were measured over 20-60 epochs, spanning $\sim 100$ days during 1993-95. Such spectra display intensity scintillation patterns that fade over narrow frequency ranges and short time intervals (Fig. 1), arising from diffractive scintillation effects. We obtained dynamic spectra data with a frequency resolution $\approx 140 \mathrm{kHz}$ and a time resolution $\sim 10$ secs.

To quantify the average characteristics of scintillation patterns at any epoch, we have computed the two-dimensional auto co-variance function 

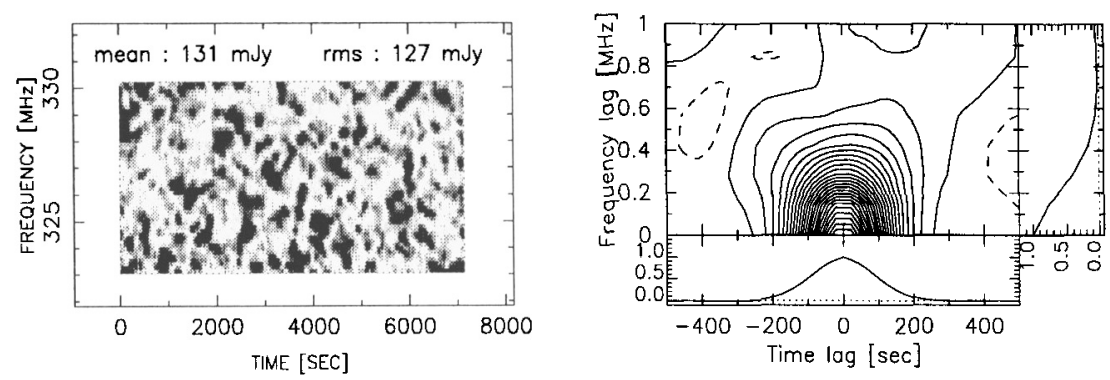

Fig. 1. The dynamic scintillation spectrum of PSR B0919+06 as observed on 24 May 1994, darker areas representing higher intensity values (left panel). The ACF is shown along with $1 \mathrm{D}$ cuts across zero lags of frequency and time (right panel).

(ACF) and fitted a two-dimensional gaussian to yield decorrelation bandwidth $\nu_{d}$ and scintillation time scale $\tau_{d}$, which are the widths of the ACF along zero time and frequency lag axes respectively (Gupta et al. 1994). The large number of epochs of observations allow us to average out long-term fluctuations of these parameters, arising from refractive scintillation effects, giving an accuracy of $5-10 \%$. From these estimates, we derive the line-ofsight averaged strength of scattering $\overline{C_{n}^{2}}$ (Cordes et al. 1985), given by

$$
\overline{C_{n}^{2}} \propto \nu_{o b s}^{\alpha} D^{-\alpha / 2} \nu_{d}^{-\left(\frac{\alpha-2}{2}\right)}
$$

where $\nu_{o b s}$ is the observing frequency, D is the distance estimate of the pulsar (based on the model given by Taylor and Cordes 1993), and $\alpha$ is the powerlaw index for electron density spectrum (Armstrong et al. 1995). We have assumed $\alpha=\frac{11}{3}$ in our calculations.

The derived values of $\overline{C_{n}^{2}}$ show roughly two orders of magnitude fluctuations (ranging from $10^{-4.8 \pm 0.02}$ to $10^{-3.1 \pm 0.02} \mathrm{~m}^{-20 / 3}$ ), which is about 10 times larger than earlier estimates and much larger than that predicted from current models for the $\overline{C_{n}^{2}}$ distribution in the galaxy (Cordes et al. 1985). Fig. 2.c shows these variations have a systematic trend, in that they are dominant for nearby ( $D \lesssim 1.5 \mathrm{kpc}$ ) pulsars. In addition to this, we find several cases where pulsars at comparable DMs or distances show remarkably different scintillation characteristics. In order to study such effects, we consider two pulsars at similar DMs, having decorrelation bandwidths $\nu_{d_{1}}$ and $\nu_{d_{2}}$, and define an anomaly parameter $\left(A_{d m}\right)$ as

$$
\left.A_{d m}=\frac{\left(\nu_{d_{1}} / \nu_{d_{2}}\right)_{\text {observed }}}{\left(\nu_{d_{1}} / \nu_{d_{2}}\right)_{\text {expected }}} \quad \text { (if } A_{d m}<1, \text { then } A_{d m}=\frac{1}{A_{d m}}\right)
$$

A plot of the anomaly parameter against DM (Fig. 2.a) shows a systematic variation, implying density fluctuations are distributed in a structure that is local and is asymmetrically located relative to the sun. The anomaly parameter as a function of the distance (Fig. 2.b) shows a similar behaviour. 

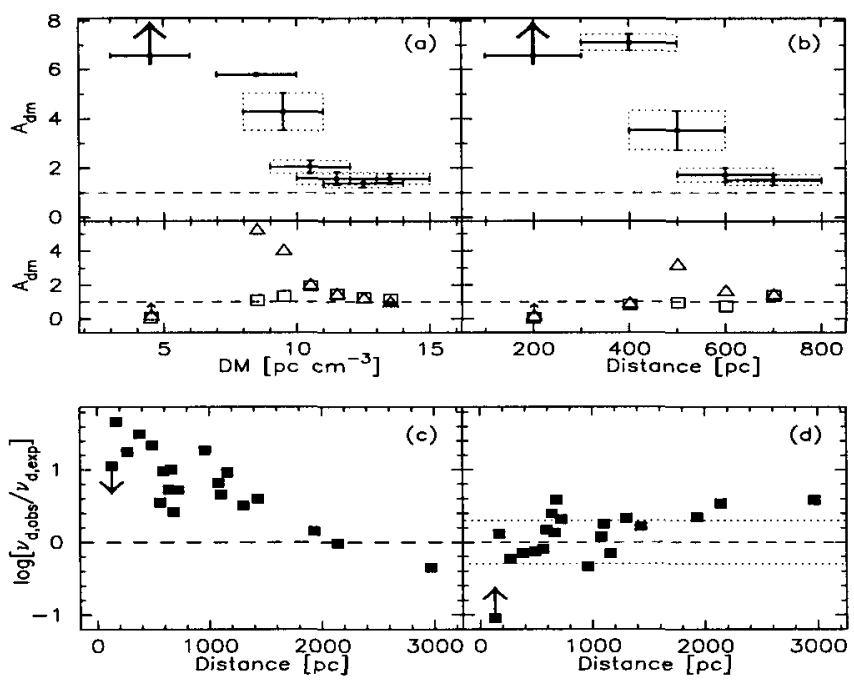

Fig. 2. The variations of the parameter $A_{d m}$ are shown with $D M$ (a) and with distance (b), where the expected values of $\nu_{d}$ are for a uniform scattering medium. The lower panels of $(a)$ and $(b)$ are for the 3-component medium ( $\Delta$ for uniform shell and $\square$ for non-uniform shell). In (c), values of $\nu_{d, e x p}$ are computed for a uniform medium and in (d), they are for the 3-component medium (non-uniform shell).

\section{Interpretation of Results}

We try to understand our observations by comparing the measured anomalies with anomalies predicted by specific density structures around the sun. Simple models consisting of an excess or deficiency of scattering material around the sun are unable to reproduce the observed trends of $\mathrm{A}_{\mathrm{dm}}$ and $\nu_{d, o b s} / \nu_{d, e x p}$. To explain our observations, we need a 3-component scattering medium with a cavity surrounded by a shell of much higher density fluctuations embedded in the normal, large scale ISM. The sun has to be located away from the centre of the cavity. By adjusting the parameters of the model, we are able to generate a reasonable agreement with the observations. By further assuming that the strength of scattering from the shell decreases with z-height, we are able to improve the agreement (lower panels of Figs. 2.a and 2.b). The best fit values of the parameters of the local scattering structure are such that the observed trends of the parameters $\mathrm{A}_{\mathrm{dm}}$ and $\nu_{d}$ (Fig. 2) are reproduced. The geometry of the structure is schematically shown in Fig. 3. For the size, constraints from our model are $a \sim 55 \mathrm{pc}, b \sim 60 \mathrm{pc}$ and $250<c<300 \mathrm{pc}$. The centre has an offset $r_{c} \sim 40 \mathrm{pc}$ from the sun, towards $210^{\circ}<l<235^{\circ}$ and $-21^{\circ}<b<21^{\circ}$. The density fluctuations are such that $10^{-5}<\overline{C_{n}^{2}}<10^{-4.45} \mathrm{~m}^{-20 / 3}$ for the inner cavity, $10^{-1}<\int_{0}^{d} C_{n}^{2}(l) d l<10^{-0.57} \mathrm{pc} \mathrm{m}^{-20 / 3}$ for the shell, (where $\mathrm{d}$ is the thickness) and $\overline{C_{n}^{2}}<10^{-3.37} \mathrm{~m}^{-20 / 3}$ for the outer ISM. Since the strength of scattering of the shell is much higher than those of the cavity and 
the outer ISM, it contributes substantially to the scattering of nearby pulsars outside the shell. The relative contribution from the shell decreases with the distance, which gives rise to systematic variations of $A_{d m}$ and $\nu_{d}$ (Fig. 2). The offset from the sun $\left(r_{c}\right)$ and the size in the galactic plane $(a$ and $b)$ are not uniquely constrained and our results can also be explained by a relatively bigger, but less asymmetric structure (the dashed geometry in Fig. 3).
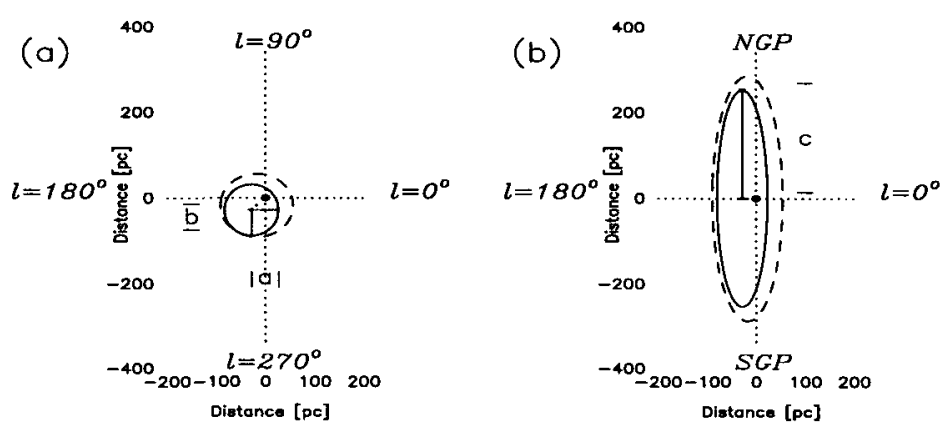

Fig. 3. The model for the local scattering structure shown as cuts through the galactic plane (a) and NGP-SGP plane (b). The solid geometry is for $r_{\mathrm{c}} \sim 40 \mathrm{pc}$, $a \sim 55 \mathrm{pc}$ and $b \sim 60 \mathrm{pc}$, and the dashed one has $r_{c} \sim 25 \mathrm{pc}$ and $a \approx b \sim 75 \mathrm{pc}$.

The exact relations between the scattering strengths and other properties of the medium are not well understood. If we assume $C_{n}^{2} \propto n_{e}^{2}$, where $n_{e}$ is the mean electron density, our observations would imply a density contrast $\sim$ 10-20 times between the shell and the ambient ISM in the case of a thin shell $(\sim 1 \mathrm{pc})$ and $\sim 5-8$ times for $\sim 10 \mathrm{pc}$ thick shell. The simple model considered here does not constrain the thickness of the shell. The pulsar PSR B0950+08 that has a parallax distance $\approx 130 \mathrm{pc}$ is located, in our model, within the cavity implying $n_{e} \approx 0.02 \mathrm{~cm}^{-3}$, which is 4 times larger than its value from $\mathrm{X}$-ray data (Snowden et al. 1990). Our constraints are, morphologically, in broad agreement with that of local bubble, obtained from X-ray, UV and HI data. Our results suggest that the bubble is surrounded by a shell of much higher density fluctuations and further investigations are required towards accurate estimates on more detailed characteristics of the shell boundary.

\section{References}

Armstrong, J.W., Rickett, B.J., Spangler, S.R. (1995): ApJ 443, 209

Cordes, J.M., Weisberg, J.M., Boriakoff, V. (1985): ApJ 288, 221

Gupta, Y., Rickett, B.J., Lyne, A.G. (1994): MNRAS 269, 1035

Rickett B.J. (1990): Ann. Rev. Astron. Ap. 28, 561

Snowden, S.L., Cox, D.P., McCammon, D., Sanders, W.T. (1990): ApJ 354, 211

Taylor, J.H., Cordes, J.M. (1993): ApJ 411, 674 